\title{
SIRTI and FOXOI mRNA expression in PBMC correlates to physical activity in COPD patients
}

\author{
This article was published in the following Dove Press journal: \\ International journal of COPD \\ 3 November 2017 \\ Number of times this article has been viewed
}

\section{Chihiro Taka \\ Ryuji Hayashi \\ Kazuki Shimokawa \\ Kotaro Tokui \\ Seisuke Okazawa \\ Kenta Kambara \\ Minehiko Inomata \\ Toru Yamada \\ Shoko Matsui \\ Kazuyuki Tobe}

First Department of Internal Medicine, Faculty of Medicine, University of Toyama, Sugitani, Toyama, Toyama, Japan
Correspondence: Ryuji Hayashi First Department of Internal Medicine, Faculty of Medicine, University of Toyama, 2630 Sugitani, Toyama, Toyama, 930-0194, Japan

$\mathrm{Tel}+8 \mathrm{I} 764347808$

Email hsayaka@med.u-toyama.ac.jp
Background: Physical activity (PA) is considered as one of the most important prognostic predictors in chronic obstructive pulmonary disease (COPD) patients. Longevity gene, SIRT1, is reported to be involved in the pathogenesis of COPD by regulating the signaling pathways of oxidative stress, inflammation, and aging. We hypothesize that SIRT1 and related genes are also associated with the benefits of PA in COPD patients.

Methods: Eighteen COPD outpatients were enrolled in this study, and their PA level was assessed with an accelerometer. We assessed the SIRT1 and related genes mRNA expression levels in the peripheral blood mononuclear cells (PBMCs) of the subjects. We carried out respiratory function testing, blood gas analysis, the 6-minute walk test, and measurement of the cross-sectional area of the erector spinae muscles (ESMCSA) by chest computed tomography. We analyzed the association of PA with the results of each of the examinations.

Results: The mean age was $72 \pm 9$ years, and the mean forced expiratory volume in 1 second was $1.4 \pm 0.56 \mathrm{~L}(52 \% \pm 19 \%$ predicted $)$. Our findings revealed a correlation between the daily PA and ESMCSA. The SIRT1 and Forkhead box O (FOXO) 1 mRNA expression levels in PBMCs were positively correlated with moderate-PA time $(r=0.60, p=0.008$ for SIRT1 and $r=0.59, p=0.01$ for $F O X O 1$ ).

Keywords: COPD, accelerometer, mRNA, walking, sedentary, moderate

\section{Introduction}

Chronic obstructive pulmonary disease (COPD) is one of the leading causes of chronic respiratory failure. Its prevalence is about $10 \%$ in the adult population $>40$ years of age in many countries. ${ }^{1}$ Long-term exposure to cigarette smoke, which is a major cause of COPD, induces chronic inflammation in the airways and lungs, resulting in airway remodeling and obstructive pulmonary dysfunction. COPD is also considered to be a systemic disease. The gaseous and noxious particles such as cigarette smoke can cause oxidative stress and induce systemic inflammation in individual patients. ${ }^{2}$ While certain pharmacological therapies can ameliorate the symptoms of COPD, this disease is generally progressive and ultimately leads to death caused by respiratory failure or systemic comorbidities. ${ }^{3}$ New therapeutic strategy is therefore urgently needed.

Recently, physical activity (PA) has been suggested as a useful predictor of the all-cause mortality in patients with $\mathrm{COPD}^{4}$ and guidelines on the management of COPD recommend increase in the PA levels of these patients. ${ }^{5}$ However, the physiological mechanisms by which PA affects the outcomes of COPD are unknown. If those mechanisms were precisely recognized, the control of COPD patients would be improved.

SIRT1 is a nicotinamide adenine dinucleotide dependent histone/protein deacetylase, mainly localized in the cell nucleus, ${ }^{6,7}$ and has drawn a lot of attention as a mediator of longevity. In COPD, it has been reported that SIRT1 plays a central role in the 
cellular responses to oxidative stress, cellular senescence, and regulation of inflammation by deacetylating certain substrates such as Forkhead box O proteins (FOXOs), p53, and nuclear factor- $\kappa \mathrm{B} .{ }^{8}$ Therefore, SIRT1 is expected to prevent the progression of the disease..$^{9}$ In addition, exercise has been reported to increase the mRNA levels and protein expression of SIRT1, as well as the activity of this enzyme in the skeletal muscle ${ }^{10,11}$ and caloric restriction increased SIRT1 in peripheral blood mononuclear cells (PBMCs). ${ }^{12}$

FOXOs function as homeostasis regulators and coordinate responses to environmental changes including oxidative stress. ${ }^{13}$ The FOXO conserved family consists of four members, FOXO1, FOXO3, FOXO4, and FOXO6. SIRT1 and $\mathrm{FOXO1}$ are regulated with positive feedback mechanisms in gene expression. ${ }^{14}$ It was reported that FOXO1 expression was increased in the atrophic muscle of COPD patients. ${ }^{15}$ Because FOXO1 target genes include antioxidant enzymes such as super oxide dismutase 2 and catalase, it is probable that SIRT1 and FOXO1 have a protective effect in oxidative environments such as COPD.

It is complex and difficult to reveal the mechanisms of PA's beneficial effects on COPD prognosis. Kao et al reported that the mRNA expression levels of SIRT1, FOXO1, and $F O X O 3$ in blood samples obtained from patients with chronic dizziness were repressed as compared with those in the samples obtained from healthy people. ${ }^{16}$ They also showed that exercise could upregulate the SIRT1 expression level in PBMCs. Therefore, we hypothesized that there might be a correlation between the PA level and the expression of SIRT1 or FOXOs in COPD patients. To explore this hypothesis, we designed this cross-sectional study and found the correlation between them.

\section{Materials and methods Study design and participants}

A total of 18 male COPD outpatients examined between June 2015 and March 2016 were included in this cross-sectional observational study conducted at Toyama University Hospital. In all the subjects, COPD had been diagnosed based on Global initiative for chronic Obstructive Lung Disease (GOLD). ${ }^{17}$ They were receiving treatment with inhaled drugs such as a long-acting muscarinic antagonist (LAMA) or long-acting $\beta 2$ agonist (LABA). All the subjects were stable for 3 months prior to the study start. From each of the subjects, we collected data on clinical variables and the average of daily PA level. The study was conducted with the approval of the Ethics Committee of Toyama University Hospital (Clinical 27-8), and written informed consent was obtained from each of the subjects.

\section{Clinical variables}

Body mass index was calculated from weight and height of the patients measured at the time of the respiratory function testing. Smoking history was obtained from the medical records. The forced vital capacity (FVC) and forced expiratory volume in 1 second $\left(\mathrm{FEV}_{1}\right)$ were measured by spirometry (CHESTAC-8900; CHEST, Tokyo, Japan) in conformity with the American Thoracic Society (ATS) recommendations. ${ }^{18}$ Blood gas analysis was performed in each subject under room air. Exercise tolerance was assessed by measuring the 6-minute walking distance (6MWD) in accordance with the ATS guidelines. ${ }^{19}$ The erector spinae muscles' cross-sectional area (ESMCSA) was measured using axial chest computed tomographic (CT) images according to a previously described method with some modifications. ${ }^{20,21}$ We identified the erector spinae muscles at the level of the lower margin of the 12th thoracic vertebra and manually drew a line surrounding it; the ESMCSA was presented as the sum of the bilateral sectional areas calculated by the chest CT software (SYNAPSE; Fujifilm, Tokyo, Japan).

\section{PA assessment}

The PA level was assessed by a triaxial accelerometer (Active style Pro HJA-750C; OMRON HEALTHCARE Co, Ltd, Tokyo, Japan), which calculated metabolic equivalents (METs) by its own algorithm distinguishing exercise from daily activity. ${ }^{22}$ For 2 weeks, patients were instructed to wear the accelerometer on their waist belts upon awaking except for the bath time. ${ }^{23}$ The collected data were analyzed by a dedicated accessory software. To analyze PA, in addition to the total energy expenditure (TEE) and PA-related energy expenditure (PAEE) (kcal/day), we evaluated the time length spent in every intensity level of activity ( $\mathrm{min} /$ day). ${ }^{24}$ In this study, PA was classified into several intensity levels according to the sensitivity of the accelerometer: sedentary, 1-1.9 METs; light-intensity PA, 2-2.9 METs; moderateintensity PA, 3-5.9 METs; vigorous PA, $\geq 6$ METs. ${ }^{25}$ All the measurements were expressed as the daily mean.

\section{Measurement of the mRNA expression levels of SIRTI, FOXOI, FOXO3, and p53}

Peripheral blood samples from each subject were collected into vacutainer tubes containing ethylenediaminetetraacetic acid dipotassium salt as the anticoagulant. PBMCs were isolated from the whole blood samples within 2 hours of sample collection by Ficoll-Hypaque (Ficoll-Paque PLUS; GE Healthcare Bio-Sciences AB, Uppsala, Sweden) gradient centrifugation ${ }^{26,27}$ and immediately preserved in a $-80^{\circ} \mathrm{C}$ 
freezer until the assay. ${ }^{12}$ Total RNA was extracted from the PBMCs using an RNA extracting reagent (ISOGEN; Nippon Gene Co, Ltd, Tokyo, Japan) in accordance with the manufacturer's instruction. The concentration of the total RNA was measured by spectrophotometry (Nano Drop; Thermo Fisher Scientific, Waltham, MA, USA). ${ }^{12}$ After each sample was diluted homogeneously by addition of sterile distilled water, first-strand complementary DNA was synthesized using a reverse-transcription polymerase chain reaction (RT-PCR) kit (RR036A;Takara Bio Inc, Shiga, Japan) in accordance with the manufacturer's instructions. Quantitative RT-PCR was performed using the MX3000p Real-Time PCR System (Agilent Technologies, Santa Clara, CA, USA) to determine the mRNA expression levels of SIRT1, FOXO1, FOXO3, p53, and glyceraldehyde-3-phosphate dehydrogenase (GAPDH). Quantitative RT-PCR for each gene was performed using the TaqMan method $\left(95^{\circ} \mathrm{C}\right.$ for 10 minutes, and 50 cycles of $95^{\circ} \mathrm{C}$ for 15 seconds and $60^{\circ} \mathrm{C}$ for 1 minute) with Premade Primer sets (SIRT1: Hs01009005_m1, FOXO1: Hs01054576_m1, FOXO3: Hs00818121_m1, p53: Hs01034249_m1, GAPDH: Hs03929097_g1; Thermo Fisher Scientific). All reactions were performed in duplicate and expression levels of the target mRNAs were normalized by the GAPDH expression level.

\section{Statistical analysis}

The obtained data are expressed as mean \pm standard deviation. Comparison of continuous data was performed by Student's $t$-test. To analyze the correlations between parameters, Pearson's correlation coefficient was used in case of normal distribution data and Spearman's rank correlation coefficient was used for non-normal distribution data. All statistical analyses were performed using the JMP pro 11 software (SAS Institute Inc, Cary, NC, USA), and the statistical significance level was set at $p<0.05$.

\section{Results}

\section{Patient characteristics}

Eighteen COPD patients participated in this study and are described according to GOLD stage 1-2 or 3-4 (Table 1). The mean age of stage 3-4 patients was significantly less than that of stage 1-2 patients. All subjects had a smoking history. The mean arterial oxygen tension under room air of stage 1-2 patients was significantly higher than that of stage 3-4 patients. The mean of 6MWD was around $340 \mathrm{~m}$ in both groups. The mean of ESMCSA was not different in both groups. A percentage of stage 3-4 patients were treated with LABA and LAMA and/or long-term oxygen therapy. No stage 1-2 patients received either treatment.
Table I Subjects characteristics $(n=18)$

\begin{tabular}{|c|c|c|c|}
\hline \multirow{2}{*}{$\begin{array}{l}\text { Variables/ } \\
\text { treatment }\end{array}$} & \multicolumn{2}{|c|}{ GOLD stage } & \multirow[t]{2}{*}{$p$-value } \\
\hline & $\mathrm{I}-2(\mathrm{n}=9)$ & $3-4(n=9)$ & \\
\hline Age, years & $75 \pm 2.9$ & $68.1 \pm 2.9$ & 0.036 \\
\hline $\mathrm{BMI}, \mathrm{kg} / \mathrm{m}^{2}$ & $20.0 \pm 2.53$ & $22.6 \pm 2.75$ & 0.059 \\
\hline Smoking history (\%) & $9(100)$ & $9(100)$ & \\
\hline Pack-years & $56.5 \pm 23.0$ & $59.9 \pm 20.8$ & 0.75 \\
\hline FVC, L & $3.18 \pm 0.86$ & $2.83 \pm 0.44$ & 0.15 \\
\hline FVC, \% predicted & $95.1 \pm 17.4$ & $81.0 \pm 12.8$ & 0.034 \\
\hline $\mathrm{FEV}_{1}, \mathrm{~L}$ & $\mid .78 \pm 0.5 \mathrm{I}$ & $1.06 \pm 0.33$ & 0.0013 \\
\hline $\mathrm{FEV}_{1}, \%$ predicted & $67.2 \pm 11.4$ & $37.4 \pm 10.7$ & $<0.000$ I \\
\hline $\mathrm{PaO}_{2}$, Torr ${ }^{\mathrm{a}}$ & $91.5 \pm 10.9$ & $78.0 \pm 7.17$ & 0.012 \\
\hline 6MWD, $\mathrm{m}^{\mathrm{b}}$ & $334 \pm 110$ & $344 \pm 106$ & 0.57 \\
\hline ESMCSA, mm² & $2,394 \pm I, 494$ & $2,984 \pm 546$ & 0.28 \\
\hline \multicolumn{4}{|l|}{ Current inhaled drugs } \\
\hline LAMA & $4(44)$ & $7(78)$ & \\
\hline LABA & $2(22)$ & $5(56)$ & \\
\hline LABA + LAMA & $0(0)$ & $4(44)$ & \\
\hline LTOT administered & $0(0)$ & $3(33)$ & \\
\hline
\end{tabular}

Notes: Data are expressed as mean \pm SD or number (\%). $p$-values were calculated by Student's $t$-test. $\mathrm{PaO}_{2}$ is the arterial oxygen tension at rest under room air condition. ${ }^{a} \mathrm{n}=13,{ }^{\mathrm{b}} \mathrm{n}=17$.

Abbreviations: 6MWD, 6-minute walk distance; ESMCSA, cross-sectional area of erector spinae muscles; LABA, long-acting $\beta 2$ agonist; LAMA, long-acting muscarinic antagonist; LTOT, long-term oxygen therapy; SD, standard deviation; GOLD, Global initiative for chronic Obstructive Lung Disease; FVC, forced vital capacity; FEV forced expiratory volume in I second; BMI, body mass index.

\section{Measurement of PA}

Because of the small sample size, we analyzed all the data together. The mean duration of wearing the accelerometer by the patients was $728 \pm 72 \mathrm{~min} /$ day. The mean PAEE was $452 \pm 127 \mathrm{kcal} /$ day and the mean TEE was $1,931 \pm 240$ (Table 2). The mean duration of sedentary posture was $578 \pm 70 \mathrm{~min} /$ day. The mean time engaged in light-intensity PA, moderate-intensity PA, and vigorous PA was $113 \pm 43$, $37 \pm 24$, and $0.5 \pm 0.5 \mathrm{~min} /$ day, respectively (Figure 1). The sedentary time accounted for a large part of the daily lives of the patient and little time was spent in vigorous activity.

\section{Relationship between clinical variables and PA level}

The relationships between the clinical variables and PA levels were evaluated (Table 3). Age was negatively correlated

Table 2 Measurements of physical activity assessed by accelerometer $(n=18)$

\begin{tabular}{ll}
\hline Measurements & Values \\
\hline Analyzed days, days & $9.6 \pm 4.8$ \\
Wearing time, min/day & $728 \pm 72$ \\
TEE, kcal/day & $1,931 \pm 240$ \\
PAEE, kcal/day & $452 \pm 127$ \\
\hline
\end{tabular}

Note: Data are expressed as mean \pm SD.

Abbreviations: TEE, total energy expenditure; PAEE, physical activity-related energy expenditure; SD, standard deviation. 


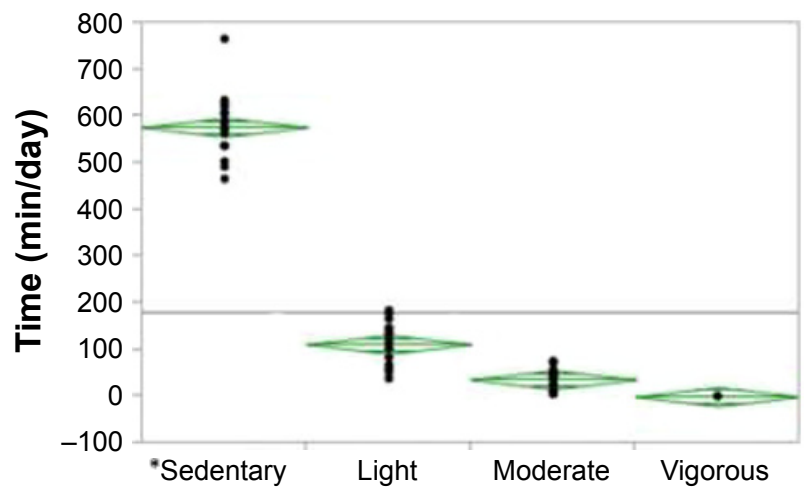

Figure I Time spent in various PA intensities.

Notes: Sedentary is I-1.9 METs; light is 2-2.9 METs; moderate is 3-5.9 METs; and vigorous is $\geq 6$ METs.

Abbreviations: PA, physical activity; METs, metabolic equivalents.

with PAEE ( $r=-0.51, p=0.031)$. FVC \% predicted and $\mathrm{FEV}_{1} \%$ were negatively correlated with sedentary time ( $r=-0.53, p=0.02$ and $r=-0.55, p=0.02$, respectively). ESMCSA was positively correlated with PAEE $(r=0.72$, $p<0.001)$.

\section{mRNA gene expression and PA level}

Because $p 53$ gene is reported to be regulated by SIRT $1,{ }^{28}$ we measured the mRNA expression level of SIRT1, FOXOs, and $p 53$ in PBMCs. There was some relationship between the SIRT1 expression and related genes (Figure 2). We evaluated the relationship between PA level and the SIRT1 and other genes mRNA expression. The SIRT1 mRNA and FOXO1 mRNA expressions in PBMCs were positively correlated with moderate-PA time $(r=0.60$, $p=0.008$ and $r=0.59, p=0.01$, respectively) and the ratio of moderate-PA time to sedentary time $(r=0.65, p=0.003$ and $r=0.59, p=0.03$, respectively) (Figure $3 \mathrm{~A}$ and B). FOXO3 and $p 53 \mathrm{mRNA}$ expressions were not correlated to PA level (Figure 3C and D).

\section{mRNA gene expression and GOLD stage}

We compared each mRNA gene expression and GOLD stage (1-2 and 3-4). None of the genes showed any correlation to GOLD stage class (Figure 4).

\section{Discussion}

This study showed the relationship between daily PA level and SIRT1, FOXO1 gene expression in COPD patients. To the best of our knowledge, this is the first report suggesting the mechanism of PA's benefit on good prognosis in COPD patients.

PA is defined as any bodily movement produced by skeletal muscles that causes energy expenditure in excess of basal metabolism. ${ }^{4}$ In this study, the accelerometer was used for assessment of the average daily PA level in the subjects. Because the precise quantification of PA can be difficult, ${ }^{25}$ we compared the level of PA to several examinations which could be related to PA to confirm our method precision. Our results revealed that $\mathrm{FEV}_{1} \%$ predicted was correlated with the PA level, and the 6MWD also tended to be correlated. The correlation between ESMCSA and PA level in this study suggests that a higher PA level can maintain more muscle volume. These results suggest that the PA level measured with the accelerometer in this study precisely reflected the daily PA level of the subjects.

Although PA is the best prognostic predictor for COPD, the precise mechanisms of its role are largely unknown. It was reported that PA was restricted due to dyspnea and fatigue on exertion and a sedentary lifestyle might lead to physical deconditioning in COPD patients. ${ }^{25}$ Rajendrasozhan et al showed decrease of the SIRT1 mRNA expression level in the lungs of COPD patients. ${ }^{29}$ Nakamaru et al also showed that SIRT1 mRNA expression level was significantly lower in the lung tissue and PBMCs of patients with severe COPD as compared to those in nonsmoking control subjects. ${ }^{30}$

Table 3 Relationship between clinical variables and the physical activity level $(n=18)$

\begin{tabular}{|c|c|c|c|c|c|c|c|c|}
\hline \multirow[t]{2}{*}{ Variables } & \multicolumn{2}{|l|}{$\underline{\text { PAEE }}$} & \multicolumn{2}{|c|}{ Sedentary } & \multicolumn{2}{|c|}{ Moderate } & \multicolumn{2}{|c|}{ Moderate/sedentary } \\
\hline & $\boldsymbol{r}$ & $p$-value & $\boldsymbol{r}$ & $p$-value & $r$ & $p$-value & $r$ & $p$-value \\
\hline Age, years & $-0.5 \mathrm{I}$ & 0.031 & -0.09 & 0.73 & -0.37 & 0.13 & -0.40 & 0.10 \\
\hline $\mathrm{BMI}, \mathrm{kg} / \mathrm{m}^{2}$ & 0.23 & 0.37 & 0.38 & 0.12 & -0.24 & 0.34 & -0.23 & 0.36 \\
\hline FVC, \% predicted & -0.08 & 0.75 & -0.53 & 0.02 & 0.13 & 0.60 & 0.20 & 0.44 \\
\hline $\mathrm{FEV}_{1}, \%$ predicted & -0.17 & 0.49 & -0.55 & 0.02 & -0.06 & 0.82 & -0.04 & 0.89 \\
\hline $\mathrm{PaO}_{2}$, Torr & -0.37 & 0.21 & -0.29 & 0.34 & -0.46 & 0.11 & -0.39 & 0.19 \\
\hline 6MWD, m & -0.03 & 0.91 & -0.30 & 0.25 & 0.22 & 0.39 & 0.36 & 0.16 \\
\hline ESMCSA, $\mathrm{mm}^{2}$ & 0.72 & $<0.001$ & 0.02 & 0.92 & 0.19 & 0.45 & 0.32 & 0.20 \\
\hline
\end{tabular}

Notes: $r$ is the correlation coefficient; $\mathrm{PaO}_{2}$ is the arterial oxygen tension at rest under room air condition. Data are expressed by Pearson's correlation coefficient except FVC \% predicted. Spearman's rank correlation coefficient was used in case of FVC \% predicted. aModerate/sedentary was calculated as the ratio of the moderate physical activity time to sedentary time.

Abbreviations: PAEE, physical activity-related energy expenditure; 6MWD, 6-minute walk distance; ESMCSA, cross-sectional area of erector spinae muscles; FVC, forced vital capacity; $\mathrm{FEV}_{1}$, forced expiratory volume in I second; BMI, body mass index. 

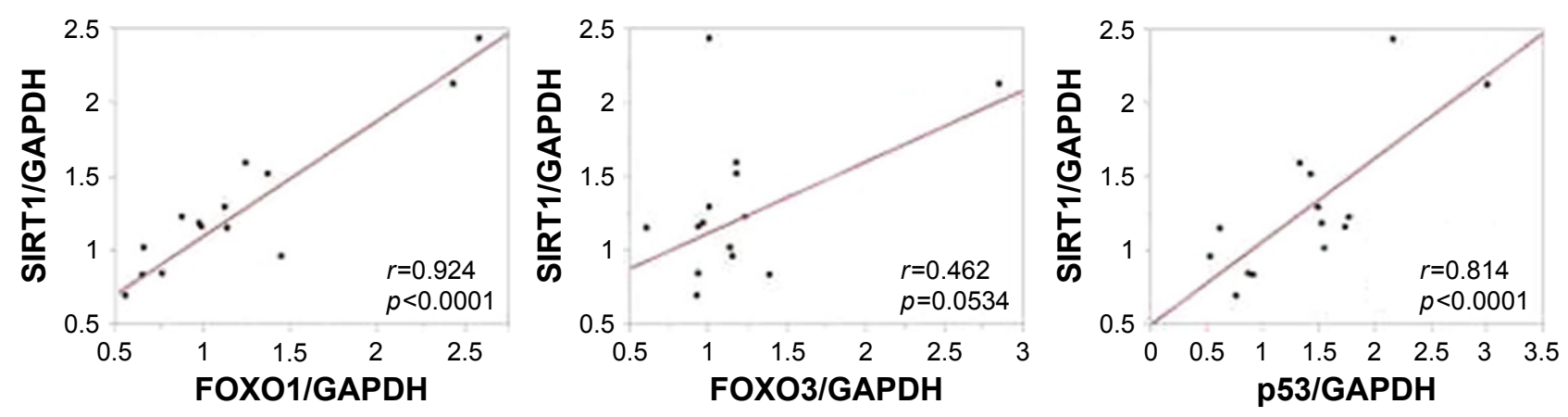

Figure 2 The relationship between SIRTI mRNA expression and related genes in PBMC.

Notes: The expression level of the target mRNAs in PBMC was normalized to GAPDH expression level. All reactions were performed in duplicate. The correlation between SIRTI and FOXOI, FOXO3, or p53 was calculated and the data are expressed by Spearman's rank correlation coefficient. $r$ is the correlation coefficient.

Abbreviations: PBMC, peripheral blood mononuclear cell; GAPDH, glyceraldehyde-3-phosphate dehydrogenase; FOXO, Forkhead box O.

As for the relationship of PA and SIRT1/FOXO1, exercise or fasting is reported to promote SIRT1 and FOXO activity for energy expenditure. ${ }^{31}$ Tobacco smoke or noxious gas inhalation causes $\mathrm{COPD}^{2}$ and oxidative burden is reported to be increased in peripheral blood ${ }^{32}$ and may cause detrimental effects in COPD patients. Because SIRT1 and FOXO1 together has an antioxidant effect, ${ }^{33}$ decreased SIRT1 expression in COPD patients might lead to excessive
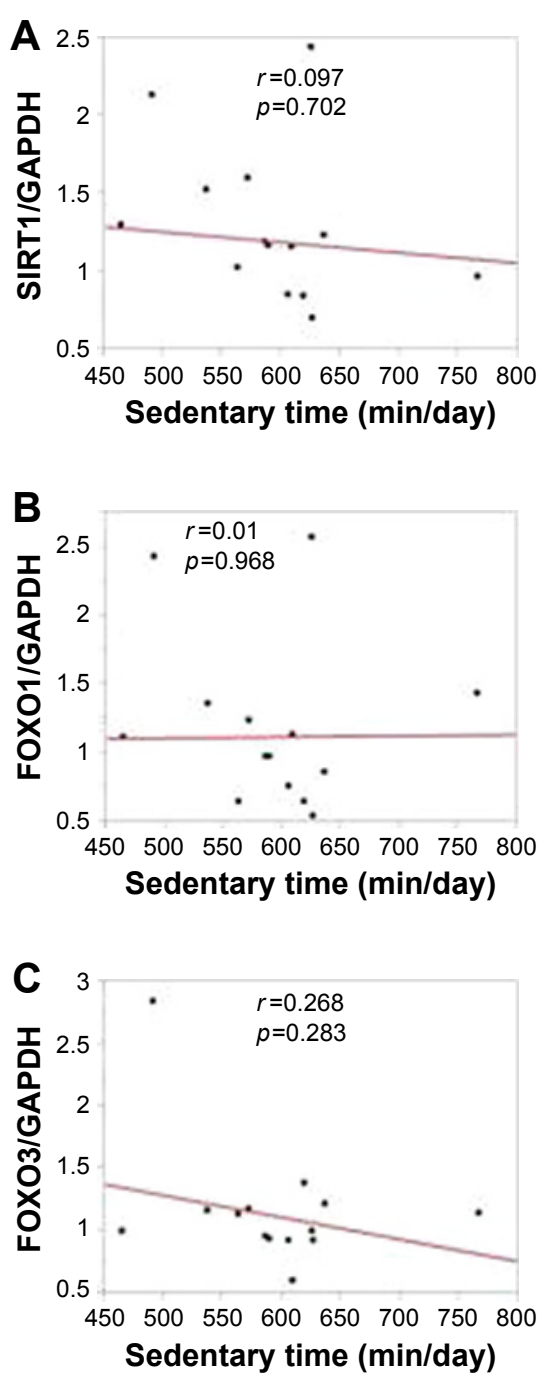
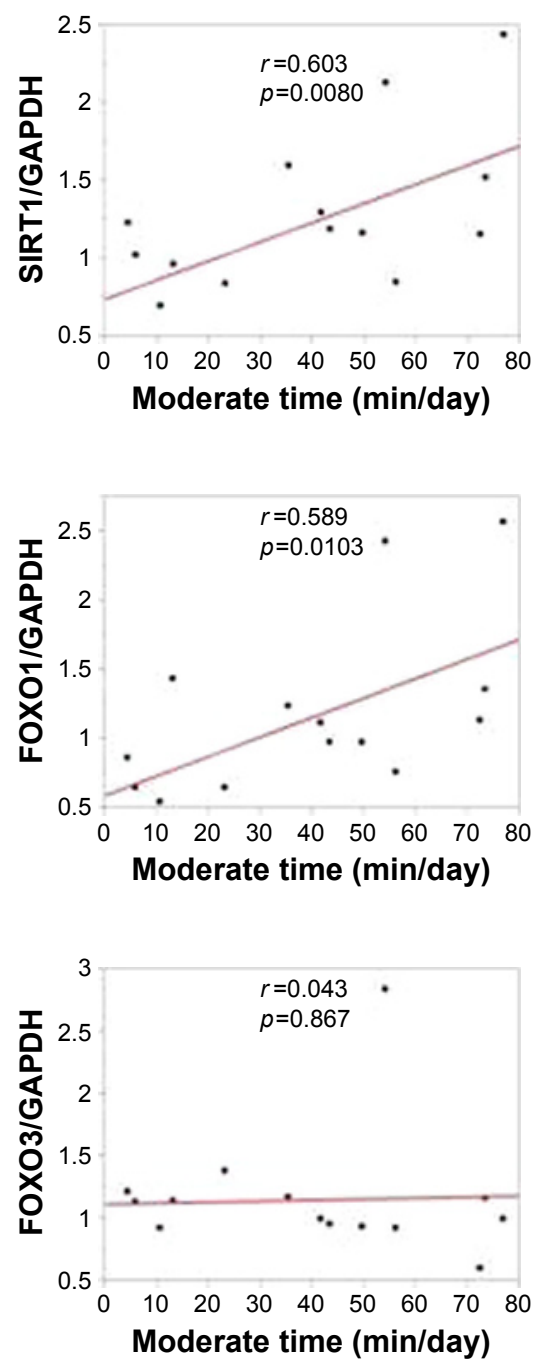
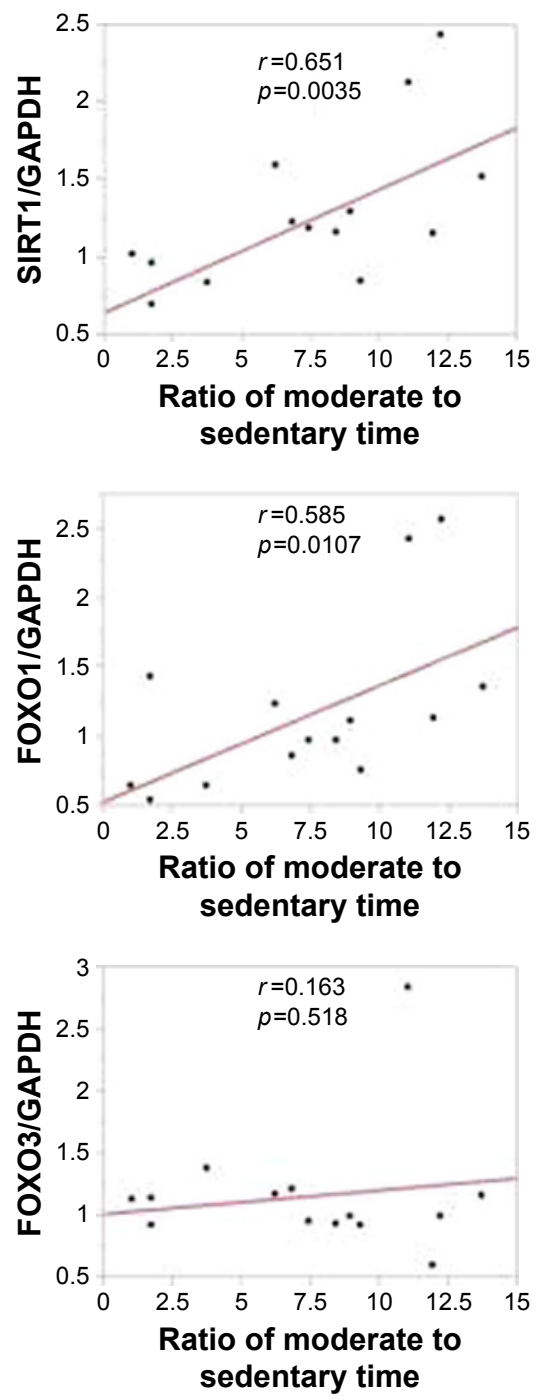

Figure 3 (Continued) 

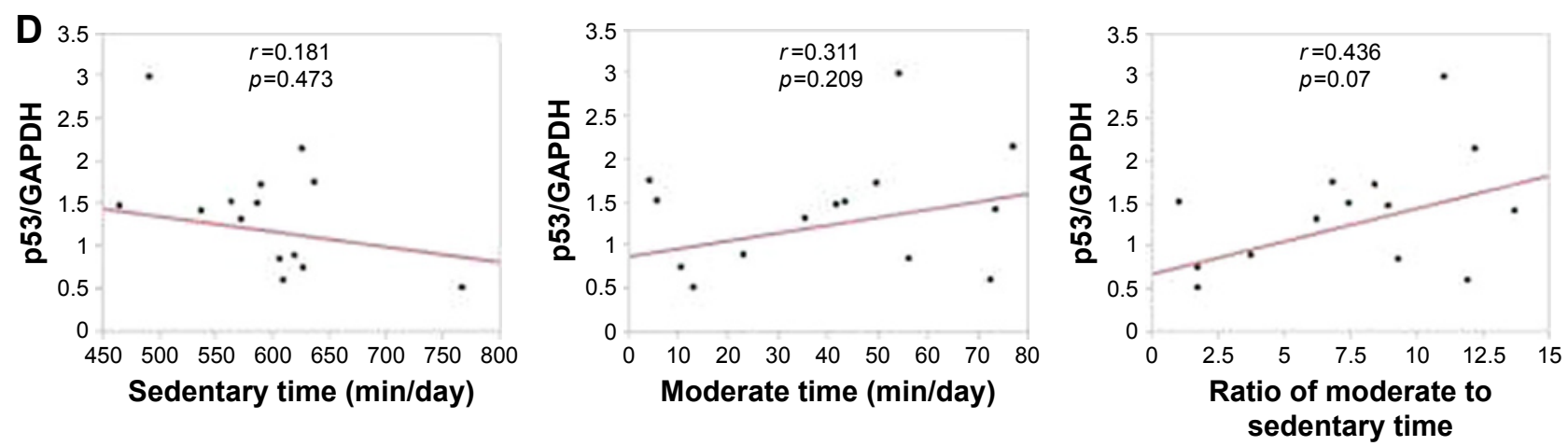

Figure 3 The relationship between gene expressions in PBMC and sedentary or moderate physical activity level. Note: (A) SIRTI mRNA, (B) FOXOI mRNA, (C) FOXO3 mRNA, (D) p53 mRNA.

Abbreviations: PBMC, peripheral blood mononuclear cell; GAPDH, glyceraldehyde-3-phosphate dehydrogenase; FOXO, Forkhead box O.

oxidative stress. In addition, SIRT1 is considered to have a protective effect against COPD and to play an important role in the pathogenesis of COPD. ${ }^{34,35}$ All these reports prompted us to consider that PA might benefit COPD patients through induction of SIRT1 and FOXO1 against oxidative stress. Our results support the hypothesis that PA promotes induction of SIRT1 and FOXO1 mRNA expression and contributes to an antioxidant effect in COPD patients. Although p53 mRNA expression was correlated to SIRT1, it was not correlated to the PA level. p53 and SIRT1 were reported to have opposite effects on each other. ${ }^{36}$ p53 may play a different role from SIRT1 in COPD patients.

We did not find the relationship between any gene expression and TEE or PAEE (data not shown). On the other hand,
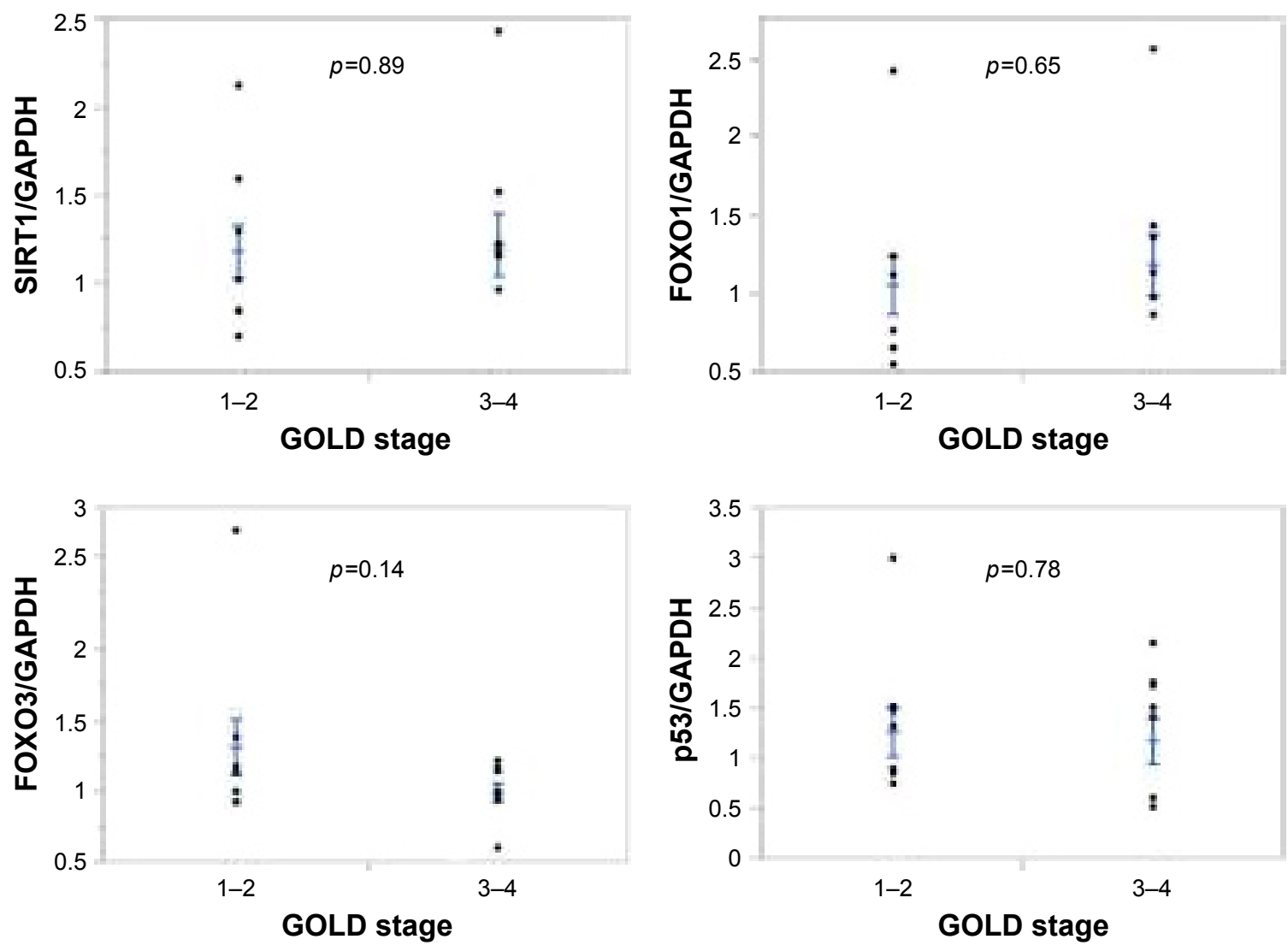

Figure 4 Comparisons of each gene expression in PBMC according to GOLD stage.

Notes: Comparisons of GOLD stage classes were performed by Student's $t$-test. Bars shows mean and standard error of the mean.

Abbreviations: GOLD, Global Initiative for chronic Obstructive Lung Disease; PBMC, peripheral blood mononuclear cell; GAPDH, glyceraldehyde-3-phosphate dehydrogenase; FOXO, Forkhead box O. 
we found that the ratio of moderate-PA time to sedentary time was strongly correlated with SIRT1 and FOXO1 expression level. These results suggest that decreased sedentary time is also important for regulation of SIRT1 and FOXO1 mRNA expression. PA was classified as light, moderate, or vigorous in accordance with the level of energy expenditure. ${ }^{25}$ Recently, it was suggested that increasing the time spent in moderate or vigorous PA leads to improvement of the all-cause mortality ${ }^{37}$ and prevention of lifestyle-related diseases and cancer. ${ }^{5}$ In contrast, a sedentary lifestyle increases the risk of mortality. ${ }^{38}$ In line with these reports, our study results suggested the importance of decreased sedentary time for SIRT1 and FOXO1 expression. On the other hand, no correlation was found between any gene expression and GOLD stage class. Especially, SIRT1 and FOXO1 gene expression results were different from the case of PA. SIRT1 and $\mathrm{FOXO1}$ gene expressions in PBMC are not related to COPD stage class (severity) but are correlated to PA. This may be an important result for the management of COPD patients because COPD patients of any GOLD stage class may benefit from PA.

Because this was a cross-sectional study, the longitudinal relationship between PA and the expression of SIRT1 and FOXO1 remains unclear. Although it is reasonable to consider that moderate PA induces SIRT1 and FOXO1 gene expression against oxidative stress in COPD patients, the precise mechanisms should be elucidated.

We did not identify the cell types inducing SIRT1 and FOXO1 gene expression in PBMC. Major cell types of PBMCs are monocyte and lymphocyte. These cell types may affect the immune regulation in COPD patients. Our future plans are to investigate which type of cells contributes to SIRT1 and FOXO1 induction and to investigate the role of those genes in the specific cell type.

Although the sample size of this study was small, a significant correlation between the PA level and PBMCs SIRT1 and FOXO1 mRNA expression was found in COPD patients. Future research on the mechanisms underlying the correlation between SIRT1/FOXO1 expression level and PA level may lead to novel management strategies for COPD patients.

\section{Acknowledgments}

We gratefully acknowledge the participation of the patients in this study. We would like to thank all involved parties in the implementation of this study. We thank Ms Yamamoto for her excellent technical help. We thank Dr Hiroaki Tanaka and Dr Toshiro Miwa for their contributions to acquisition of data.
The current affiliation of Ryuji Hayashi is Clinical Oncology, Toyama University Hospital, 2630 Sugitani, Toyama, Toyama, 930-0194, Japan.

\section{Disclosure}

The authors report no conflicts of interest in this work.

\section{References}

1. Buist AS, McBurnie MA, Vollmer WM, et al. International variation in the prevalence of COPD (the BOLD study): a population-based prevalence study. Lancet. 2007;370(9589):741-750.

2. Barnes PJ, Celli BR. Systemic manifestations and comorbidities of COPD. Eur Respir J. 2009;33(5):1165-1185.

3. Decramer M, Janssens W, Miravitlles M. Chronic obstructive pulmonary disease. Lancet. 2012;379(9823):1341-1351.

4. Waschki B, Kirsten A, Holz O, et al. Physical activity is the strongest predictor of all-cause mortality in patients with COPD: a prospective cohort study. Chest. 2011;140(2):331-342.

5. Watz H, Pitta F, Rochester CL, et al. An official European Respiratory Society statement on physical activity in COPD. Eur Respir $J$. 2014;44(6):1521-1537.

6. Fusco S, Maulucci G, Pani G. Sirt1: def-eating senescence? Cell Cycle. 2012;11(22):4135-4146.

7. Pucci B, Villanova L, Sansone L, et al. Sirtuins: the molecular basis of beneficial effects of physical activity. Intern Emerg Med. 2013; 8(Suppl 1):S23-S25.

8. Yao H, Rahman I. Perspectives on translational and therapeutic aspects of SIRT1 in inflammaging and senescence. Biochem Pharmacol. 2012; 84(10):1332-1339.

9. Lavu S, Boss O, Elliott PJ, Lambert PD. Sirtuins - novel therapeutic targets to treat age-associated diseases. Nat Rev Drug Discov. 2008; 7(10):841-853.

10. Pardo PS, Boriek AM. The physiological roles of Sirt1 in skeletal muscle. Aging (Albany NY). 2011;3(4):430-437.

11. Oliveira NR, Marques SO, Luciano TF, et al. Treadmill training increases SIRT-1 and PGC-1 alpha protein levels and AMPK phosphorylation in quadriceps of middle-aged rats in an intensity-dependent manner. Mediators Inflamm. 2014;2014:987017.

12. Crujeiras AB, Parra D, Goyenechea E, Martinez JA. Sirtuin gene expression in human mononuclear cells is modulated by caloric restriction Eur J Clin Invest. 2008;38(9):672-678.

13. Eijkelenboom A, Burgering BM. FOXOs: signalling integrators for homeostasis maintenance. Nat Rev Mol Cell Biol. 2013;14(2):83-97.

14. Xiong S, Salazar G, Patrushev N, Alexander RW. FOXO1 mediates an autofeedback loop regulating SIRT1 expression. J Biol Chem. 2011; 286(7):5289-5299.

15. Pomies P, Blaquiere M, Maury J, Mercier J, Gouzi F, Hayot M. Involvement of the FoxO1/MuRF1/Atrogin-1 signaling pathway in the oxidative stress-induced atrophy of cultured chronic obstructive pulmonary disease myotubes. PloS One. 2016;11(8):e0160092.

16. Kao CL, Tsai KL, Cheng YY, Kuo CH, Lee SD, Chan RC. Vestibular rehabilitation ameliorates chronic dizziness through the SIRT1 axis. Front Aging Neurosci. 2014;6:27.

17. Rabe KF, Hurd S, Anzueto A, et al. Global strategy for the diagnosis, management, and prevention of chronic obstructive pulmonary disease: GOLD executive summary. Am J Respir Crit Care Med. 2007;176(6): $532-555$.

18. Miller MR, Hankinson J, Brusasco V, et al. Standardisation of spirometry. Eur Respir J. 2005;26(2):319-338.

19. Holland AE, Spruit MA, Troosters T, et al. An official European Respiratory Society/American Thoracic Society technical standard: field walking tests in chronic respiratory disease. Eur Respir J. 2014;44(6): $1428-1446$. 
20. Tanimura K, Sato S, Fuseya Y, et al. Quantitative assessment of erector spinae muscles in patients with chronic obstructive pulmonary disease. Novel chest computed tomography-derived index for prognosis. Ann Am Thorac Soc. 2016;13(3):334-341.

21. Lee CS, Cron DC, Terjimanian MN, et al. Dorsal muscle group area and surgical outcomes in liver transplantation. Clin Transplant. 2014;28(10):1092-1098.

22. Oshima Y, Kawaguchi K, Tanaka S, et al. Classifying household and locomotive activities using a triaxial accelerometer. Gait Posture. 2010;31(3):370-374.

23. Kawagoshi A, Kiyokawa N, Sugawara K, et al. Effects of low-intensity exercise and home-based pulmonary rehabilitation with pedometer feedback on physical activity in elderly patients with chronic obstructive pulmonary disease. Respir Med. 2015;109(3):364-371.

24. Nakayama M, Bando M, Araki K, et al. Physical activity in patients with idiopathic pulmonary fibrosis. Respirology. 2015;20(4):640-646.

25. Hill K, Gardiner PA, Cavalheri V, Jenkins SC, Healy GN. Physical activity and sedentary behaviour: applying lessons to chronic obstructive pulmonary disease. Intern Med J. 2015;45(5):474-482.

26. Lee H, Chu SH, Park JY, Park HK, Im JA, Lee JW. Visceral adiposity is associated with SIRT1 expression in peripheral blood mononuclear cells: a pilot study. Endocr J. 2013;60(11):1269-1273.

27. Godfrin-Valnet M, Khan KA, Guillot X, et al. Sirtuin 1 activity in peripheral blood mononuclear cells of patients with osteoporosis. Med Sci Monit Basic Res. 2014;20:142-145.

28. Hori YS, Kuno A, Hosoda R, Horio Y. Regulation of FOXOs and p53 by SIRT1 modulators under oxidative stress. PloS One. 2013;8(9): e73875.

29. Rajendrasozhan S, Yang SR, Kinnula VL, Rahman I. SIRT1, an antiinflammatory and antiaging protein, is decreased in lungs of patients with chronic obstructive pulmonary disease. Am J Respir Crit Care Med. 2008;177(8):861-870.
30. Nakamaru Y, Vuppusetty C, Wada $H$, et al. A protein deacetylase SIRT1 is a negative regulator of metalloproteinase-9. FASEB J. 2009; 23(9):2810-2819.

31. Canto C, Gerhart-Hines Z, Feige JN, et al. AMPK regulates energy expenditure by modulating NAD+ metabolism and SIRT1 activity. Nature. 2009;458(7241):1056-1060.

32. Can U, Yerlikaya FH, Yosunkaya S. Role of oxidative stress and serum lipid levels in stable chronic obstructive pulmonary disease. J Chin Med Assoc. 2015;78(12):702-708.

33. Sin TK, Yu AP, Yung BY, et al. Modulating effect of SIRT1 activation induced by resveratrol on Foxo1-associated apoptotic signalling in senescent heart. J Physiol. 2014;592(12):2535-2548.

34. Chun P. Erratum to: role of sirtuins in chronic obstructive pulmonary disease. Arch Pharm Res. 2015;38(5):932.

35. Rahman I, Kinnula VL, Gorbunova V, Yao H. SIRT1 as a therapeutic target in inflammaging of the pulmonary disease. Prev Med. 2012; 54(Suppl):S20-S28.

36. Nakazawa H, Chang K, Shinozaki S, et al. iNOS as a driver of inflammation and apoptosis in mouse skeletal muscle after burn injury: possible involvement of Sirt1 S-nitrosylation-mediated acetylation of p65 NF-kappaB and p53. PLoS One. 2017;12(1):e0170391.

37. Manini TM, Everhart JE, Patel KV, et al. Daily activity energy expenditure and mortality among older adults. JAMA. 2006;296(2):171-179.

38. Biswas A, Oh PI, Faulkner GE, et al. Sedentary time and its association with risk for disease incidence, mortality, and hospitalization in adults: a systematic review and meta-analysis. Ann Intern Med. 2015; 162(2):123-132.
International Journal of COPD

\section{Publish your work in this journal}

The International Journal of COPD is an international, peer-reviewed journal of therapeutics and pharmacology focusing on concise rapid reporting of clinical studies and reviews in COPD. Special focus is given to the pathophysiological processes underlying the disease, intervention programs, patient focused education, and self management protocols.

\section{Dovepress}

This journal is indexed on PubMed Central, MedLine and CAS. The manuscript management system is completely online and includes a very quick and fair peer-review system, which is all easy to use. Visit http://www.dovepress.com/testimonials.php to read real quotes from published authors. 